\title{
Kinetic-theory approach to low-energy collective modes in nuclei
}

\author{
V. Abrosimov ${ }^{\mathrm{a}}$, A.Dellafiore ${ }^{\mathrm{b}}$ and F.Matera ${ }^{\mathrm{b}}$ \\ ${ }^{a}$ Institute for Nuclear Research, 252028 Kiev, Ukraine \\ b Istituto Nazionale di Fisica Nucleare and Dipartimento di Fisica, \\ Universita' di Firenze, Largo E.Fermi 2, 50125 Firenze, Italy
}

\begin{abstract}
Two different solutions of the linearized Vlasov equation for finite systems, characterized by fixed and moving-surface boundary conditions, are discussed in a unified perspective. A condition determining the eigenfrequencies of collective nuclear oscillations, that can be obtained from the moving-surface solution, is studied for isoscalar vibrations of lowest multipolarity. Analytic expressions for the friction and mass parameters related to the low-enegy surface excitations are derived and their value is compared to values given by other models. Both similarities and differences are found with respect to the other approaches, however the close agreement obtained in many cases with one of the other models suggests that, in spite of some important differences, the two approaches are substantially equivalent. The formalism based on the Vlasov equation is more transparent since it leads to analytical expressions that can be a basis for further improvement of the model.
\end{abstract}

PACS: 24.10.Cn, 24.30.Gd

Keywords: Vlasov equation, moving surface, collective excitations. 


\section{INTRODUCTION}

A collisionless kinetic equation with time-dependent mean field has been used long ago, first by Vlasov and then by Landau, to describe plasma oscillations in the high-frequency regime [1]. More recently Bertsch has proposed to use the same equation to study smallamplitude oscillations in nuclei [2]. The approach of Vlasov and Landau assumes translation invariance of the system under study, extensions of their approach to finite systems have to face the nontrivial problem of which boundary conditions to impose on the fluctuation of the sigle-particle density distribution $\delta n(\mathbf{r}, \mathbf{p}, t)$.

A solution of the linearized Vlasov equation suitable for describing giant resonances of various multipolarities in heavy spherical nuclei has been derived in [3]. The boundary conditions employed in [3] are inspired by the Steinwedel-Jensen (SJ) model of the giant dipole resonance (GDR) (compressible fluid within fixed boundary, see for example Ref. [4, p.558). In Ref. [5] an approach similar to that of Ref. [3] has been followed, but with boundary conditions that are inspired by the Goldhaber-Teller (GT), rather than by the SJ model. In the GT model of the GDR the neutron and proton fluids are assumed to oscillate against each other without being compressed (see for example Ref. 《4, p. 558).

In order to adopt such a description, the authors of [5] had to account for the reflection of nucleons on the moving nuclear suface (assumed to be sharp), and this was achieved by modifying the boundary conditions employed in [3].

In this paper we first recall the approaches of Refs. [3] and [5], giving a unified discussion of the different solutions derived in those papers. Then we consider the condition determining the eigenfrequencies of collective modes that has been derived in Ref. [5]. Since in that paper this condition has been studied in detail only for monopole $(L=0)$ oscillations, we turn our attention to the lowest isoscalar modes with $L>0$. We introduce a low-frequency approximation that allows us to obtain analytical expressions for the dynamic coefficients (friction and mass parameters) entering the equation of motion for the surface oscillations of a system of fermions. These coefficients and the associated eigenfrequencies of collective oscillations are then compared to the analogous expressions given by the liquid-drop model (LDM) of Ref. [6] and by a more closely related model discussed in Ref. [7]

\section{ONE-DIMENSIONAL EXAMPLE}

In Ref. [3] it has been shown that for a spherical system the solution of the linearized Vlasov equation can be reduced to the solution of a one-dimensional problem in the effective potential $U_{0}(r)+\frac{\lambda^{2}}{2 m r^{2}}$.

In this section we briefly compare the approaches of [3] and [5] by assuming a onedimensional model, our aim is to make the connection between the two approaches more transparent. Thus we consider a one-dimensional system described by an equilibrium Hamiltonian

$$
h_{0}(x)=\epsilon=\frac{p^{2}}{2 m}+U_{0}(x)
$$

The (possibly self-consistent) equilibrium mean field $U_{0}(x)$ is assumed to have the shape of a potential well, so that the motion of particles is bounded within two classical turning 
points $x_{1(2)}$ defined by $U_{0}\left(x_{1(2)}\right)=\epsilon$. In the Hartree approximation the relation between the self-consistent field and the interaction between particles $V\left(\left|x-x^{\prime}\right|\right)$ is

$$
U_{0}(x)=\int d x^{\prime} d p^{\prime} V\left(\left|x-x^{\prime}\right|\right) n_{0}\left(x^{\prime}, p^{\prime}\right)
$$

with $n_{0}(x, p)$ the single-particle phase-space distribution at equilibrium. We assume that at time $t=0$ this system is acted upon by a weak external field of the type $\delta(t) Q(x)$ which induces a time-dependent fluctuation of the equilibrium single-particle distribution: $n_{0}(x, p) \rightarrow n_{0}(x, p)+\delta n(x, p, t)$. As a consequence the equilibrium mean field also acquires a time-dependence: $U_{0}(x) \rightarrow U_{0}(x)+\delta U(x, t)$. It is convenient to take the Fourier transform with respect to time and to change variables from $(x, p)$ to $(x, \epsilon)$, so that

$$
\delta n(x, \epsilon, \omega)=\delta n_{+}(x, \epsilon, \omega)+\delta n_{-}(x, \epsilon, \omega),
$$

with $\delta n_{ \pm}(x, \epsilon, \omega)=\delta n\left(x, p= \pm \sqrt{2 m\left[\epsilon-U_{0}(x)\right]}, \omega\right)$. Then, according to [3], the linearized Vlasov equation implies

$$
\begin{aligned}
& \frac{\partial \delta n_{+}(x, \epsilon, \omega)}{\partial x}-A(x, \epsilon, \omega) \delta n_{+}(x, \epsilon, \omega)=B_{+}(x, \epsilon, \omega) \\
& \frac{\partial \delta n_{-}(x, \epsilon, \omega)}{\partial x}+A(x, \epsilon, \omega) \delta n_{-}(x, \epsilon, \omega)=B_{-}(x, \epsilon, \omega)
\end{aligned}
$$

with

$$
A(x, \epsilon, \omega)=\frac{i \omega}{\sqrt{\frac{2}{m}\left[\epsilon-U_{0}(x)\right]}}
$$

and

$$
B_{ \pm}(x, \epsilon, \omega)=\left(\frac{\partial n_{0}}{\partial \epsilon}\right)\left[\frac{d Q(x)}{d x}+\frac{\partial \delta U(x, \omega)}{\partial x}\right] .
$$

The solution of the system (2.4) is not completely trivial because the fluctuation of the mean field

$$
\delta U(x, \omega)=\int d x^{\prime} d p^{\prime} V\left(\left|x-x^{\prime}\right|\right)\left[\delta n_{+}\left(x^{\prime}, \epsilon^{\prime}, \omega\right)+\delta n_{-}\left(x^{\prime}, \epsilon^{\prime}, \omega\right)\right]
$$

couples the two equations. In the approach of [3] the solution is obtained in two steps, first the mean field fluctuation in (2.6) is neglected, thus obtaining a zero-order solution $\delta n_{ \pm}^{0}$ that can be derived explicitly, then the solution with the full inhomogeneous term (2.6) is expressed in implicit form through an integral equation similar to that given by the random-phase approximation (RPA) for the particle-hole Green function.

Of course the zero-order solution $\delta n_{ \pm}^{0}$ depends on the boundary conditions. The conditions employed in [3] were

$$
\delta n_{+}\left(x_{1(2)}, \epsilon, \omega\right)=\delta n_{-}\left(x_{1(2)}, \epsilon, \omega\right) .
$$

In this case the solution of the system (2.4) (neglecting mean-field fluctuations) becomes 


$$
\delta n_{ \pm}^{0}(x, \epsilon, \omega)=e^{ \pm i \omega \tau(x)}\left[\int_{x_{1}}^{x} d x^{\prime} B^{0}\left(x^{\prime}, \epsilon\right) e^{\mp i \omega \tau\left(x^{\prime}\right)}+C(\omega)\right],
$$

with $B^{0}(x, \epsilon)=\left(\frac{\partial n_{0}}{\partial \epsilon}\right)\left[\frac{d Q}{d x}\right]$

$$
\begin{gathered}
\tau(x)=\int_{x_{1}}^{x} d y \frac{1}{\sqrt{\frac{2}{m}\left[\epsilon-U_{0}(y)\right]}}, \\
C(\omega)=\frac{e^{i \omega T} \int_{x_{1}}^{x_{2}} d x^{\prime} B^{0}\left(x^{\prime}, \epsilon\right) e^{-i \omega \tau\left(x^{\prime}\right)}-\int_{x_{1}}^{x_{2}} d x^{\prime} B^{0}\left(x^{\prime}, \epsilon\right) e^{i \omega \tau\left(x^{\prime}\right)}}{1-e^{i \omega T}}, \\
T=2 \tau\left(x_{2}\right) .
\end{gathered}
$$

(We have simply re-written in more compact form the solution given in Sect.4 of [3]. Note that there is a misprint in Eq.(4.13b) of [3], that should read: $\left.C_{2}=\frac{C_{r}-C_{l} \exp (-i \omega T / 2)}{1-\exp (i \omega T)}\right)$.

In Ref. [5] it has been argued that the effects of moving boundary can be taken into account, at least in a linearized approximation, simply by changing the boundary conditions (2.8). In particular, since we are interested in the radial motion of nucleons in the effective potential $U_{0}(r)+\frac{\lambda^{2}}{m r^{2}}$, it is sufficient to change the boundary condition at one turning point (say $x_{2}$ ). Thus, instead of the boundary conditions (2.8), in [5] the following boundary conditions have been used

$$
\begin{aligned}
\delta n_{+}\left(x_{1}, \epsilon, \omega\right) & =\delta n_{-}\left(x_{1}, \epsilon, \omega\right) \\
\delta n_{+}\left(x_{2}, \epsilon, \omega\right) & =\delta n_{-}\left(x_{2}, \epsilon, \omega\right)+\left(\frac{\partial n_{0}}{\partial \epsilon}\right) F(\omega),
\end{aligned}
$$

where $F(\omega)$ is the Fourier transform of an as yet undetermined function of time $F(t)$.

The solution analogous to (2.9) with these new boundary conditions is

$$
\delta \tilde{n}^{0}=\delta n^{0}+\delta n_{S}^{0}
$$

with

$$
\delta n_{S}^{0}(x, \epsilon, \omega)=\left(\frac{\partial n_{0}}{\partial \epsilon}\right) F(\omega) \frac{\cos [\omega \tau(x)]}{i \sin (\omega T / 2)} .
$$

We can obtain $\delta n^{0}(t)$ by taking the inverse Fourier transform of $\delta n^{0}(\omega)$

$$
\delta n^{0}(t)=\frac{1}{2 \pi} \int_{-\infty}^{+\infty} d \omega e^{-i \omega t} \delta n^{0}(\omega)
$$

which can be determined by contour integration in the complex $\omega$-plane. Since we are interested in $\delta n^{0}(t)$ for $t>0$, the integration contour must be closed in the lower part of the complex plane. The first term on the r.h.s. of Eq. (2.9) is analytic in the whole plane, and the only contributions to the integral come from the poles of $C(\omega)$ that are at

$$
\omega=n \frac{2 \pi}{T}-i \eta \quad\left(n \text { integer, } \eta \rightarrow 0^{+}\right)
$$

where $1-e^{i \omega T}=0$. Equivalently we can thik of $\omega$ as a complex variable having a small positive imaginary part $i \eta$. The moving-boundary solution is more complicated since the pole structure of $\delta n_{S}^{0}(\omega)$ is not as simple as that of $\delta n^{0}(\omega)$. Apart from the usual poles at $\omega=n \frac{2 \pi}{T}$, the quantity $\delta n_{S}^{0}(\omega)$ can have additional poles due to $F(\omega)$, and these extra poles may contribute to the contour integral. The problem of determining the explicit form of $F(t)$, and consequently the pole structure of $F(\omega)$, will be discussed in the next section. 


\section{THREE-DIMENSIONAL (SPHERICAL) CASE}

In this section we recall the approach followed in Ref. [5] in order to determine the pole structure of the additional term in the boundary condition (2.13).

The approach of [5] is inspired by the LDM [6], the nuclear mean field is approximated by a square-well type potential, but, contrary to Ref. [3], in this model the external field is allowed to change also the equilibrium shape of the nucleus. The change of shape is parametrized as $R(\theta, \varphi, t)=R+\delta R(\theta, \varphi, t)$, with

$$
\delta R(\theta, \varphi, t)=\sum_{L M} \delta R_{L M}(t) Y_{L M}(\theta, \varphi) .
$$

In the LDM a surface deformation of this kind generates a fluctuation of the pressure at $r=R$ that is given by (cf. Eq.(6A-57) of [6])

$$
\left.\delta P(\mathbf{r}, t)\right|_{r=R}=\sum_{L M} C_{L} \frac{\delta R_{L M}(t)}{R^{4}} Y_{L M}(\theta, \varphi) .
$$

The restoring force parameter $C_{L}$ used in [5] contains only the contribution of the surface energy, so

$$
C_{L} \approx\left(C_{L}\right)_{\text {surf }}=\sigma R^{2}(L-1)(L+2)
$$

and $\sigma$ is the surface tension parameter that can be obtained from the mass formula:

$$
\sigma \approx 1 \mathrm{MeV} \mathrm{fm}^{-2}
$$

In Ref. [5] the link with kinetic theory was established by putting

$$
\left.\delta P(\mathbf{r}, t)\right|_{r=R}=\left.\int d \mathbf{p} p_{r} v_{r}\left[\delta n(\mathbf{r}, \mathbf{p}, t)-\left(\frac{\partial n_{0}}{\partial \epsilon}\right) \delta U(\mathbf{r}, t)\right]\right|_{r=R} .
$$

This is a kind of self-consistency condition and its physical meaning is that the nuclear surface is required to behave as a free surface. The integral on the r.h.s. is the radial-radial element of the momentum flux tensor $\delta \Pi_{r r}(\mathbf{r}, \omega)$ for the nuclear quantum liquid [8]. The radial velocity $v_{r}(r)$ is

$$
v_{r}(r)=\sqrt{\frac{2}{m}\left[\epsilon-U_{0}(r)-\frac{\lambda^{2}}{2 m r^{2}}\right]},
$$

while the radial momentum is $p_{r}=m v_{r}$.

Combining Eqs.(3.2) and (3.5), and taking the Fourier transform with respect to time gives

$$
\sigma R^{-2} \sum_{L M}(L-1)(L+2) \delta R_{L M}(\omega) Y_{L M}(\hat{\mathbf{r}})=\left.\delta \Pi_{r r}(\mathbf{r}, \omega)\right|_{r=R}
$$

This equation can be used to determine the poles of the function $F(\omega)$ appearing in the three-

dimensional generalization of Eq.(2.13), that is, the eigenfrequencies of collective nuclear 
vibrations. Multiplying both sides of Eq. (3.7) by $Y_{L M}^{*}(\hat{\mathbf{r}})$ and integrating over solid angle gives

$$
\sigma R^{-2}(L-1)(L+2) \delta R_{L M}=\left.\delta \Pi_{r r}^{L M}(r, \omega)\right|_{r=R} .
$$

The r.h.s. is the multipole component of the integral in Eq.(3.5), it is given by a generalization of Eq.(B.6) of [3]:

$$
\begin{aligned}
\delta \Pi_{r r}^{L M}(r, \omega) & =\frac{(4 \pi)^{2}}{(2 L+1)} \frac{1}{2 r^{2}} \int d \epsilon \int d \lambda \lambda p_{r} \\
& \left\{\sum_{N=-L}^{L}\left[\delta n_{+}(L M N, \epsilon \lambda r, \omega)+\delta n_{-}(L M N, \epsilon \lambda r, \omega)\right] Y_{L N}^{*}\left(\frac{\pi}{2}, \frac{\pi}{2}\right)\right. \\
& \left.-\left(\frac{\partial n_{0}}{\partial \epsilon}\right) \frac{2 L+1}{4 \pi} \delta U_{L M}(r, \omega)\right\} .
\end{aligned}
$$
3

The quantities $\delta n_{ \pm}(L M N, \epsilon \lambda r, \omega)$ satisfy the system of differential equations (2.4) with

$$
A(N, \epsilon \lambda r, \omega)=\frac{i \omega}{v_{r}(r)}-i \frac{N}{v_{r}(r)} \frac{\lambda}{m r^{2}}
$$

replacing Eq.(2.5)and (there are some misprints in Eq.(5.12 of [3])

$$
B_{ \pm}(L M N, \epsilon \lambda r, \omega)=\left(\frac{\partial n_{0}}{\partial \epsilon}\right)\left[\left(\frac{\partial}{\partial r} \pm i \frac{N}{v_{r}(r)} \frac{\lambda}{m r^{2}}\right)\left(Q_{L M}(r)+\delta U_{L M}(r, \omega)\right)\right] Y_{L N}\left(\frac{\pi}{2}, \frac{\pi}{2}\right)
$$

instead of Eq.(2.6). Like in the one-dimensional example, the solution of the linearized Vlasov equation (2.4) can be studied in two steps. At first we can neglect, both in Eqs.(3.9) and (3.11), the mean-field fluctuation $\delta U_{L M}$ that couples the two equations, thus obtaining a zero-order approximation that may be a starting point for deriving the more complete solution that takes into account $\delta U_{L M}$. In this paper we investigate only the zero-order approximation and show that even at this level some interesting results about low-energy isoscalar collective modes can be obtained.

The zero-order solution analogous to Eq. 2.9) (fixed surface) is

$$
\begin{aligned}
\delta n_{ \pm}^{0}(L M N, \epsilon \lambda r, \omega) & =e^{ \pm i[\omega \tau(r)-N \gamma(r)]}\left[\int_{r_{1}}^{r} d r^{\prime} B_{ \pm}^{0}\left(L M N, \epsilon \lambda r^{\prime}\right) e^{\mp\left[i \omega \tau\left(r^{\prime}\right)-N \gamma\left(r^{\prime}\right)\right]}\right. \\
& +C(L M N, \epsilon \lambda, \omega)]
\end{aligned}
$$

with

$$
\begin{aligned}
B_{ \pm}^{0}(L M N, \epsilon \lambda r) & =\left(\frac{\partial n_{0}}{\partial \epsilon}\right)\left[\frac{d Q_{L M}}{d r} \pm i \frac{N}{v_{r}(r)} \frac{\lambda}{m r^{2}} Q_{L M}\right] Y_{L N}\left(\frac{\pi}{2} \frac{\pi}{2}\right) \\
\tau(r) & =\int_{r_{1}}^{r} d y \frac{1}{\sqrt{\frac{2}{m}\left[\epsilon-U_{0}(y)-\frac{\lambda^{2}}{2 m y^{2}}\right]}}
\end{aligned}
$$




$$
\begin{gathered}
\gamma(r)=\int_{r_{1}}^{r} d y \frac{1}{\sqrt{\frac{2}{m}\left[\epsilon-U_{0}(y)-\frac{\lambda^{2}}{2 m y^{2}}\right.}} \frac{\lambda}{m y^{2}}, \\
C(L M N, \epsilon \lambda, \omega)=\left\{e^{i[\omega T-N \Gamma] / 2} \int_{r_{1}}^{r_{2}} d r B_{+}^{0}(L M N, \epsilon \lambda r) e^{-i[\omega \tau(r)-N \gamma(r)]}\right. \\
\left.-\int_{r_{1}}^{r_{2}} d r B_{-}^{0}(L M N, \epsilon \lambda r) e^{i[\omega \tau(r)-N \gamma(r)]}\right\}\left\{1-e^{i[\omega T-N \Gamma]}\right\}^{-1}, \\
T=2 \tau\left(r_{2}\right) \quad \Gamma=2 \gamma\left(r_{2}\right) .
\end{gathered}
$$

The solution analogous to Eq.(2.14) (moving surface) instead, reads

$$
\begin{aligned}
\delta \tilde{n}_{ \pm}^{0}(L M N, \epsilon \lambda r, \omega) & =\delta n_{ \pm}^{0}(L M N, \epsilon \lambda r, \omega) \\
& +\left(\frac{\partial n_{0}}{\partial \epsilon}\right) e^{ \pm i[\omega \tau(r)-N \gamma(r)]} F(L M N, \epsilon \lambda, \omega) \frac{1}{2 i} \frac{1}{\sin \left(\frac{\omega T-N \Gamma}{2}\right)} .
\end{aligned}
$$

One more step is necessary in order to get the final result of Ref. [5]: the functions $F(L M N, \epsilon \lambda, \omega)$ must be related to the radial velocity of the surface (cf. Eq. (3.9) of [5]) through

$$
F(L M N, \epsilon \lambda, \omega)=i \omega 2 p_{r}(\epsilon \lambda, R) \delta R_{L M}(\omega) Y_{L N}\left(\frac{\pi}{2} \frac{\pi}{2}\right) .
$$

Replacing this expression into Eq.(3.18) and recalling that $\delta n=\delta n_{+}+\delta n_{-}$, gives $\delta \tilde{n}^{0}=$ $\delta n^{0}+\delta n_{S}^{0}$, with

$$
\delta n_{S}^{0}=2\left(\frac{\partial n_{0}}{\partial \epsilon}\right) \cos [\omega \tau(r)-N \gamma(r)] \omega p_{r}(\epsilon \lambda, R) \delta R_{L M}(\omega) Y_{L N}\left(\frac{\pi}{2} \frac{\pi}{2}\right) \frac{1}{\sin \left(\frac{\omega T-N \Gamma}{2}\right)} .
$$

Combining this equation with Eqs.(3.8) and(3.9) and neglecting the mean-field fluctuation $\delta U_{L M}$ in (3.9) gives the following expression for $\delta R_{L M}(\omega)$ in zero-order approximation:

$$
\begin{aligned}
& \delta R_{L M}^{0}(\omega)= \\
& \frac{\frac{(4 \pi)^{2}}{(2 L+1)} \frac{1}{2 R^{2}} \int d \epsilon \int d \lambda \lambda p_{r} \sum_{N} \delta n^{0}(L M N, \epsilon \lambda R, \omega) Y_{L N}^{*}\left(\frac{\pi}{2}, \frac{\pi}{2}\right)}{\left.\sigma \frac{(L-1)(L+2)}{R^{2}}-\frac{\omega}{R^{2}} \frac{(4 \pi)^{2}}{(2 L+1)} \int d \epsilon\left(\frac{\partial n_{0}}{\partial \epsilon}\right) \int d \lambda \lambda p_{r}^{2} \sum_{N} \mid Y_{L N}\left(\frac{\pi}{2}\right), \frac{\pi}{2}\right)\left.\right|^{2} \cot \left(\frac{\omega T-N \Gamma}{2}\right)} .
\end{aligned}
$$

As usual we assume that $\omega$ has a small positive imaginary part so that $\omega$ in the equation above has to be interpreted as $\omega+i \eta$. The poles of $\delta R_{L M}^{0}(\omega)$ in the complex $\omega$-plane are determined by the vanishing of the denominator in Eq. (3.21). This condition can be written in more compact form as

$$
C_{L}-\chi_{L}(\omega)=0
$$

with $C_{L}$ given by Eq.(3.3) and

$$
\chi_{L}(\omega) \equiv \omega R^{2} \frac{(4 \pi)^{2}}{(2 L+1)} \int d \epsilon\left(\frac{\partial n_{0}}{\partial \epsilon}\right) \int d \lambda \lambda p_{r}^{2} \sum_{N}\left|Y_{L N}\left(\frac{\pi}{2}, \frac{\pi}{2}\right)\right|^{2} \cot \left(\frac{(\omega+i \eta) T-N \Gamma}{2}\right) .
$$

It is useful to recall that the functions $Y_{L N}\left(\frac{\pi}{2}, \frac{\pi}{2}\right)$ vanish unless $N$ has the same parity as $L$ so that the sum over $N$ involves only terms with either odd or even $N$.

Eq. (3.22) is the main result of Ref. [5] and its solution determines the frequencies of collective nuclear excitations within that approach. 


\section{COMPARISON WITH OTHER MODELS}

It is interesting to compare Eq.(3.22) with the analogous condition given by the LDM. We consider the version that allows also for compression modes [6]. Equation (6A-58) of Ref. [6] can be written as

$$
C_{L}-\omega^{2} D_{L}\left[\frac{L}{R} \frac{j_{L}\left(\frac{\omega}{u_{c}} R\right)}{\left.\frac{\partial}{\partial r} j_{L}\left(\frac{\omega}{u_{c}} r\right)\right|_{r=R}}\right]=0
$$

with $u_{c}$ the velocity of sound.

Equations (4.1) and (3.22) are coceptually similar because they both express a condition for the eigenfrequencies of collective nuclear modes. The main difference between them is that Eq. (4.1) has been obtained in a macroscopic hydrodynamic approach, while Eq.(3.22) has been derived within a microscopic kinetic-equation approach. It is thus reasonable to expect both similarities and differences between the eigenmodes determined by the two equations.

For small $\omega$ the term in square brackets in (4.1) tends to 1 and Eq.(4.1) becomes

$$
C_{L}-\omega^{2} D_{L}=0
$$

In this approximation the surface vibrations described by $\delta R_{L M}(t)$ are pure harmonic oscillations and $\delta R_{L M}(\omega)$ has two simple poles at $\omega_{L}= \pm \sqrt{\frac{C_{L}}{D_{L}}}-i \eta$.

In order to evaluate the function $\chi_{L}(\omega)$ we take $\left(\frac{\partial n_{0}}{\partial \epsilon}\right)=-\frac{4}{(2 \pi)^{3}} \delta\left(\epsilon_{F}-\epsilon\right)$, as appropriate for a gas of zero-temperature nucleons $\left(\epsilon_{F}\right.$ is the Fermi energy, we use units such that $\left.\hbar=1\right)$, Even though Eq.(3.22) is only a zero-order approximation, it is interesting since it allows us to obtain analytical expressions for the mass parameters $D_{L}$ and for the other dynamical coefficients that determine the eigenfrequencies of collective modes. Unfortunately the functions $\chi_{L}(\omega)$ are not simple, only $\chi_{L=0}$ has been evaluated explicitly for any value of $\omega$. The result derived in Ref. [5] is, in terms of the dimensionless parameter $s \equiv \frac{\omega}{\left(v_{F} / R\right)}$,

$$
\chi_{L=0}(s)=-2 \epsilon_{F} \varrho_{0} R^{3}\left\{1+6 \sum_{n=1}^{\infty}\left[\frac{1}{3}+\frac{1}{s_{n}^{2}}-\frac{1}{s_{n}^{4}} W\left(s_{n}\right)\right]\right\}
$$

with

$$
W(s)=\frac{1}{2} s \ln \left|\frac{s+1}{s-1}\right|+i \frac{\pi}{2} s \Theta(|s|-1)
$$

$\varrho_{0}=\frac{2 p_{F}^{3}}{3 \pi^{2}}$ the equilibrium density of nuclear matter and $s_{n}=s / n \pi$.

We remark that, contrary to the hydrodynamic relation (4.1) that involves only real quantities, Eq. (3.22) involves complex quantities and thus we can expect complex eigenfrequencies as solutions, in close analogy with the Landau damping phenomenon in homogeneous systems.

In this paper we want to extend the study of the functions $\chi_{L}(\omega)$, that was initiated in Ref. [5] for the monopole case, to multipolarities $L>0$. For this purpose we write Eq.(3.23) as 


$$
\chi_{L}(\omega)=P_{L}^{2}(0) \chi_{L=0}(\omega)+\mathcal{N}_{L} \sum_{N>0}^{L}\left|Y_{L N}\left(\frac{\pi}{2}, \frac{\pi}{2}\right)\right|^{2} I_{N}(\omega)
$$

with

$$
\begin{gathered}
I_{N}(\omega)=-\omega \int_{0}^{1} d \cos \alpha \cos \alpha \sin ^{2} \alpha\left[\cot z_{N}(\alpha)+\cot z_{-N}(\alpha)\right] \\
z_{N}(\alpha)=\frac{(\omega+i \eta) \sin \alpha}{v_{F} / R}+N \alpha
\end{gathered}
$$

and

$$
\mathcal{N}_{L}=\frac{2}{\pi^{2}}\left(p_{F} R\right)^{4} \frac{4 \pi}{2 L+1}
$$

The quantities $P_{L}(x)$ are Legendre polynomials of order $L$. It is useful to recall that

$$
\begin{array}{rlrl}
\frac{4 \pi}{2 L+1}\left|Y_{L 0}\left(\frac{\pi}{2}, \frac{\pi}{2}\right)\right|^{2}=P_{L}^{2}(0) & =0 & \text { odd } L \\
& =\left[\frac{L !}{2^{L}\left(\frac{L}{2} !\right)^{2}}\right]^{2} & & \text { even } L
\end{array}
$$

To derive Eq.(4.5) we have defined $\cos \alpha=\frac{\lambda}{p_{F} R}$ in Eq.(3.23), the angle $\alpha$ has the simple geometrical interpretation shown in Fig.1. It follows immediately that $p_{r}=p_{F} \sin \alpha, T=$ $\frac{2 R \sin \alpha}{v_{F}}, \Gamma=2 \alpha$.

The integrals $I_{N}$ can be complex because the integrands can have poles. In order to evaluate these integrals it is convenient to use the following trigonometric identity

$$
\cot z_{1}+\cot z_{2}=\frac{2 \sin \left(z_{1}+z_{2}\right)}{\cos \left(z_{1}-z_{2}\right)-\cos \left(z_{1}+z_{2}\right)}
$$

and to change the integration variable to $x=\sin \alpha$, so that

$$
I_{N}(\omega)=2 \omega \int_{0}^{1} d x x^{3} \frac{\sin (2 x s)}{\cos [2 x(s+i \eta)]-\cos (2 N \arcsin x)}
$$

The terms $\cos (2 N \arcsin x) \equiv y_{N}$ are just polynomials in $x^{2}: y_{1}=1-2 x^{2}, y_{2}=1-8 x^{2}+8 x^{4}$, $y_{3}=1-18 x^{2}+48 x^{4}-32 x^{6}$, and so on. The integrals $I_{N}$ can be easily evaluated for small $s$, thus we are led to study the following low-frequency expansion for the functions $\chi_{L}(\omega)$ :

$$
\chi_{L}(\omega) \approx A_{L}+i \omega \gamma_{L}+D_{L} \omega^{2} .
$$

This expansion is valid for $s<1$, that is up to excitation energies of about $46 A^{-1 / 3} \mathrm{MeV}$, however in the monopole case the upper limit of validity is $\pi$ times larger, since in that case the relevant expansion parameter is $s / \pi$, thus we can expect to describe also the monopole compression mode within this approximation.

The three coefficients $A_{L}, \gamma_{L}$ and $D_{L}$ given by the present approach can be compared with the same coefficients obtained in the hydrodynamic approximation and to those derived in 
the more closely related approach of Ref. [7]. As we shall see, some of these coefficients vanish exactly, others (the mass parameters $D_{L}$ ) may even diverge, in any case they convey non trivial information about the low-frequency behaviour of the functions $\chi_{L}(\omega)$ and $\delta R_{L M}(\omega)$.

The coefficients $A_{L}$ in Eq.(4.12) vanish for odd multipoles, while for even multipoles

$$
A_{L}=-2 \epsilon_{F} \varrho_{0} R^{3} P_{L}^{2}(0) .
$$

The nonvanishing coefficients $A_{L}$ renormalize the restoring force parameter $C_{L}$ in Eq.(3.22). The largest value of $\left|A_{L}\right|$ occurs for $L=0$ when $A_{0}=-2 \epsilon_{F} \varrho_{0} R^{3}$. For increasing (even) $L$ the coefficients $A_{L}$ tend to zero as $1 / L$, while $C_{L}$ in Eq. (3.3) increases as $L^{2}$, so this renormalization becomes negligible in the limit of large $L$. However for $L=0 A_{L}$ is much larger than $C_{L}$, in agreement with the well known LDM result that the monopole excitation is not a surface, but a compression mode (in this case the surface tension plays a negligible role). For $L=2$ the value of $A_{L}$ is comparable to that of $C_{L}$ and this leads to a reduction of the role played by the surface tension in this excitation mode too.

The friction coefficients $\gamma_{L}$ are most easily evaluated directly from Eq.(3.23) by using the pole expansion

$$
\cot z=\sum_{n=-\infty}^{+\infty} \frac{1}{z-n \pi}
$$

It is clear also from Eq. (4.4) that for $L=0$ the friction coefficient vanishes, while for $L>0$ we find

$$
\gamma_{L}=\gamma_{w f} 2 \frac{(4 \pi)^{2}}{2 L+1} \sum_{N=1}^{L} \frac{1}{N}\left|Y_{L N}\left(\frac{\pi}{2}, \frac{\pi}{2}\right)\right|^{2} \sum_{n=1}^{+\infty} \cos \alpha_{n N} \sin ^{3} \alpha_{n N} \Theta\left(\frac{\pi}{2}-\alpha_{n N}\right)
$$

with $\alpha_{n N}=\frac{n}{N} \pi$. The angles $\alpha_{n N}$ appearing in this equation are related to the nucleon trajectory in the unperturbed mean field in the way shown in Fig.1 . It is interesting to note that when the coefficient $\gamma_{L}$ is nonvanishing, it gets a contribution only from nucleons moving along closed classical trajectories. Because of the vanishing of the coefficients $Y_{L N}\left(\frac{\pi}{2}, \frac{\pi}{2}\right)$ when the parity of $N$ differs from that of $L$, for even multipoles the sum over $N$ in Eq. (4.15) effectively starts from $N=2$ and involves only even values of $N$.

For ease of comparison with Ref. [7] we have expressed our values of $\gamma_{L}$ in terms of the wall formula friction coefficient [9] $\gamma_{w f} \equiv \frac{3}{4} \varrho_{0} p_{F} R^{4}$. We find that Eq.44.15) reproduces very well the values of $\gamma_{L}$ reported in Ref. [7]. For all value reported there (up to $L=12$ ) we find perfect agreement for odd multipoles, while for even multipoles our values of $\gamma_{L}$ are systematically about $3-4 \%$ smaller than the values of [7]. This discrepancy tends to decrease with increasing $L$, and is only about $1 \%$ for $L=12$.

From Eqs.(4.5) and (4.6) we can obtain an explicit expression for the coefficients $D_{L}$ in the expansion (4.12), it reads

$$
D_{L}=\frac{1}{5} m \varrho_{0} R^{5} P_{L}^{2}(0)+12 m \varrho_{0} R^{5} \frac{4 \pi}{2 L+1} \sum_{N>0}^{L}\left|Y_{L N}\left(\frac{\pi}{2}, \frac{\pi}{2}\right)\right|^{2} I_{N}^{\prime}
$$

with 


$$
I_{N}^{\prime} \equiv \lim _{s \rightarrow 0} \int_{0}^{1} d x \frac{x^{2}}{\frac{1-\cos (2 N \arcsin x)}{x^{2}}-2 s^{2}-i \eta s} .
$$

Note that possible terms that behave as $1 / s$ resulting from the evaluation of $I_{N}^{\prime}$ should be omitted from $D_{L}$ since they have already been included in the coefficients $\gamma_{L}$ and that the sign of the imaginary part of $I_{N}^{\prime}$ depends on the sign of $s$.

The first term on the r.h.s of Eq.(4.16) is present only for even multipolarities and is the only term for $L=0$, the remaining sum over $N$ involves only even or odd values of $N$, depending on the parity of $L$.

The first few integrals $I_{N}^{\prime}$ are:

$$
\begin{gathered}
I_{1}^{\prime}=\frac{1}{6}, \\
I_{2}^{\prime}=-\frac{1}{8} \lim _{s \rightarrow 0} \ln |s|+\frac{1}{8}(2 \ln 2-1)+i \frac{\pi}{16} \operatorname{sign} s \\
I_{3}^{\prime}=-\frac{1}{64} \frac{\sqrt{3}}{3}\left(4 \sqrt{3}+\ln \frac{1+\sqrt{3} / 2}{1-\sqrt{3} / 2}\right)+i \frac{\pi}{16} \frac{\sqrt{3}}{2} \lim _{s \rightarrow 0} \frac{1}{s}
\end{gathered}
$$

For all even multipoles with $L>0$ the real parts of the integrals $I_{N}^{\prime}$ are divergent because there is a pole exactly at the upper integration limit. This divergence agrees with that found in Ref. [7]. In the present formalism it is possible to pinpoint exactly the origin of this divergence: it is generated by nucleons moving on orbits along the diameter. A peculiar feature of the present approach is that the coefficients $D_{L}$ can be complex. In this aspect our results differ radically from those of Refs. [6] and [7]. Our coefficients $D_{L}$ are real for $L=0,1$, but for $L=2$ the real part is divergent and the imaginary part is finite. Note that this does not imply that the function $\chi_{L=2}$ has a pathological behaviuor for $\omega \rightarrow 0$, since the divergence is only logarithmic.

The integral $I_{3}^{\prime}$ is also complex, however its imaginary part behaves like $1 / s$, the corresponding term in the expansion (4.12) is linear in $\omega$ and it has already been included in the coefficient $\gamma_{L}$. Thus for example for $L=3$ only the real part of $I_{N}^{\prime}$ should be included in $D_{L}$.

In Table 1 we compare the values of the dynamic parameters given by the present approach with those of Refs. [6,7] for the first few multipoles.

As already found in Ref. [5], the present approach gives the following value for the energy of breathing modes in nuclei:

$$
\hbar \omega_{L=0} \approx \hbar \sqrt{\frac{C_{0}-A_{0}}{D_{0}}} \approx 96 A^{-1 / 3} \mathrm{MeV} .
$$

This value can be compared with that of $65 A^{-1 / 3} \mathrm{MeV}$ reported in Ref. [6] for classical hydrodynamics (both numbers have been obtained by assuming the parametrization $R=$ $1.2 A^{1 / 3} \mathrm{fm}$, for this reason our value differs from the value $103 A^{-1 / 3} \mathrm{MeV}$ of Ref. [5]). We recall that the result (4.21) is based on a zero-order approximation in which mean-field 
fluctuations in the bulk have been neglected, taking into account these effects leads to [10] $\hbar \omega_{0} \approx 82 A^{-1 / 3} \mathrm{MeV}$, in reasonable agreement with experiment [11].

The $L=1$ case is the only one in which all models of Table 1 give the same results. This simply means that none of them has problems in describing centre-of-mass motion.

For $L=2$ the eigenfrequency condition (3.22) cannot have solutions corresponding to real, small, $\omega$ because the coefficient $D_{L=2}$ is complex. Thus we have to look for possible solutions in the complex $\omega$ or $s$ plane. For this puspose we write Eq.(3.23) as

$$
\chi_{L=2}(s)=3 A_{0} s\left\{\frac{1}{4} \int_{0}^{1} d x x^{3} \cot (s x)+\frac{3}{8} \int_{0}^{1} d x x^{3}[\cot (s x-2 \alpha)+\cot (s x+2 \alpha)]\right\},
$$

with $\alpha=\arcsin x$. For small $s, \cot (s x) \approx 1 / s x-s x / 3+O\left(s^{3}\right)$, and the first integral is easily evaluated. Similarly, the second integral, using the identity (4.10), becomes

$$
\begin{aligned}
& \left.\int_{0}^{1} d x x^{3}[\cot (s x-2 \alpha)+\cot (s x+2 \alpha)]\right\} \\
= & 4 s \int_{0}^{1} d x \frac{x^{2}}{8\left(x^{2}-1\right)+2 s^{2}} \quad+O\left(s^{3}\right) \\
= & -\frac{s}{2}\left[\frac{1}{2} \ln \left(1-\frac{16}{s^{2}}\right)-1\right] \quad+O\left(s^{3}\right) .
\end{aligned}
$$

Thus for complex $s$ and $|s|<1$ we have

$$
\chi_{L=2}(s)=A_{0}\left\{\frac{1}{4}-\frac{s^{2}}{20}-\frac{9}{16} s^{2}\left[\frac{1}{2} \ln \left(\frac{16}{s^{2}}-1\right)+i \frac{\pi}{2} \operatorname{sign}(\Re s)-1\right]\right\} \quad+O\left(s^{4}\right) .
$$

If we use this approximation for $\chi_{2}(s)$ the eigenfrequency condition (3.22) has no solution with $|s|<1$ in the complex $s$-plane, thus the present model, at least in its simpleminded zero-order approximation, does not seem to be adequate for describing low-frequency isoscalar quadrupole oscillations in nuclei. Calculations of the isoscalar quadrupole response performed in Ref. [12] within the same model used here (for real $\omega$ ) suggest the existence of a collective mode in a region where $|s| \approx 1$ (cf. Fig.2b of [12]). Taking into account the attractive interaction inside nuclei (that is the term $\delta U_{L M}$ in Eqs.(3.9) and (3.11)) could bring this collective mode into the region $|s|<1$, however this would require a more numerical approach and would mean loosing the insight given by the analytic expressions of the zero-order approximation.

For $L=3$ the eigenfrequency condition (3.22) in the limit of small $\omega$ becomes

$$
i \omega \gamma_{3}+D_{3} \omega^{2}=C_{3}
$$

Using the values of parameters reported in Table 1, we find that low-energy octupole oscillations are overdamped. This result might offer a qualitative explanation for the background observed in inelastic proton scattering at small angle, that has been previously studied by assuming a semi-infinite model [13, 114.

\section{SUMMARY AND CONCLUSIONS}

Different solutions of the linearized Vlasov equation for finite systems can be obtained, depending on the boundary conditions imposed on the fluctuations of the single-particle phase-space density. 
We have discussed two different solutions (with fixed and moving surface) that can be useful in studying collective nuclear excitations. In particular we have analyzed the moving-surface solution in the low-frequency limit for the first few isoscalar nuclear modes (monopole, dipole, quadrupole and octupole) and have derived interesting analytical expressions for the friction and mass parameters entering the equation of motion for the oscillations of the nuclear surface. According to our semiclassical result, in the low-frequency limit, nuclear dissipation is due only to nucleons moving along closed classical trajectories. Numerically our friction parameters are in rather good agreement with those derived in Ref. [7]. For the mass parameters our result agree only partially with those of [7], like in that case we find a divergence of the mass parameters for even multipolarity, but our parameters can be complex, with a finite imaginary part. For odd multipolarities (dipole and octupole) our mass parameters do agree with those of Ref. [7]. Thus, even though the formalism is quite different, we conclude that the approach of Ref. [5], is substantially equivalent to that of Ref. [7]. On the contrary we find several differences with respect to the classical hydrodynamic results of Ref. [6]. These differences, the most imporant being probably the Landau damping of surface oscillations, can be ascribed to the fact that the properties of a fluid of fermions are, even in a semiclassical description, different from those of a classical fluid.

\section{ACKNOWLEDGEMENTS}

V. A. acknowleges financial support and kind hospitality from INFN, Italy. 


\section{REFERENCES}

[1] L. Landau, J. Phys. U.S.S.R. 10 (1946) 25, JETP 16 (1946) 574, Collected papers of L.D. Landau,ed. D.Ter Haar (Pergamon Press, Oxford, 1965)

[2] G.F.Bertsch, Nuclear Physics with heavy ions and mesons, Les Houches,1977, ed. R.Balian, M.Rho and G.Ripka (North-Holland, Amsterdam, 1978)

[3] D.M.Brink, A.Dellafiore and M.Di Toro, Nucl. Phys A456 (1986) 205

[4] P.Ring and P.Schuck, The Nuclear Many-body Problem (Springer, New York, 1980)

[5] V. Abrosimov, M. Di Toro and V. Strutinsky, Nucl. Phys. A562 (1993) 41

[6] A.Bohr and B.R.Mottelson, Nuclear Structure, vol.2 (Benjamin, Reading, Ma, 1975) Appendix 6A

[7] S.E.Koonin and J.Randrup, Nucl. Phys. A289 (1977) 475

[8] E.M. Lifshitz and L.P.Pitaevskii, Physical Kinetics (Pergamon, Oxford, 1981), Sect. 74

[9] J. Blocki et al., Ann. Phys. (NY) 113 (1978) 330

[10] V. Abrosimov, M. Di Toro and A. Smerzi, Zeit. Phys. A 347 (1994) 161

[11] A. Van der Woude, Progr. Part. Nucl. Phys. 18 (1987) 217

[12] V.I. Abrosimov, O. I. Davidovskaja and V.M. Kolomietz, and S. Shlomo, Phys. Rev. C 57 (1998) 2342

[13] H. Esbensen and G.F. Bertsch Ann. Phys. (N.Y.) 157 (1984) 255

[14] V.I. Abrosimov and J. Randrup, Nucl. Phys. A449 (1986) 446 


\section{FIGURES}

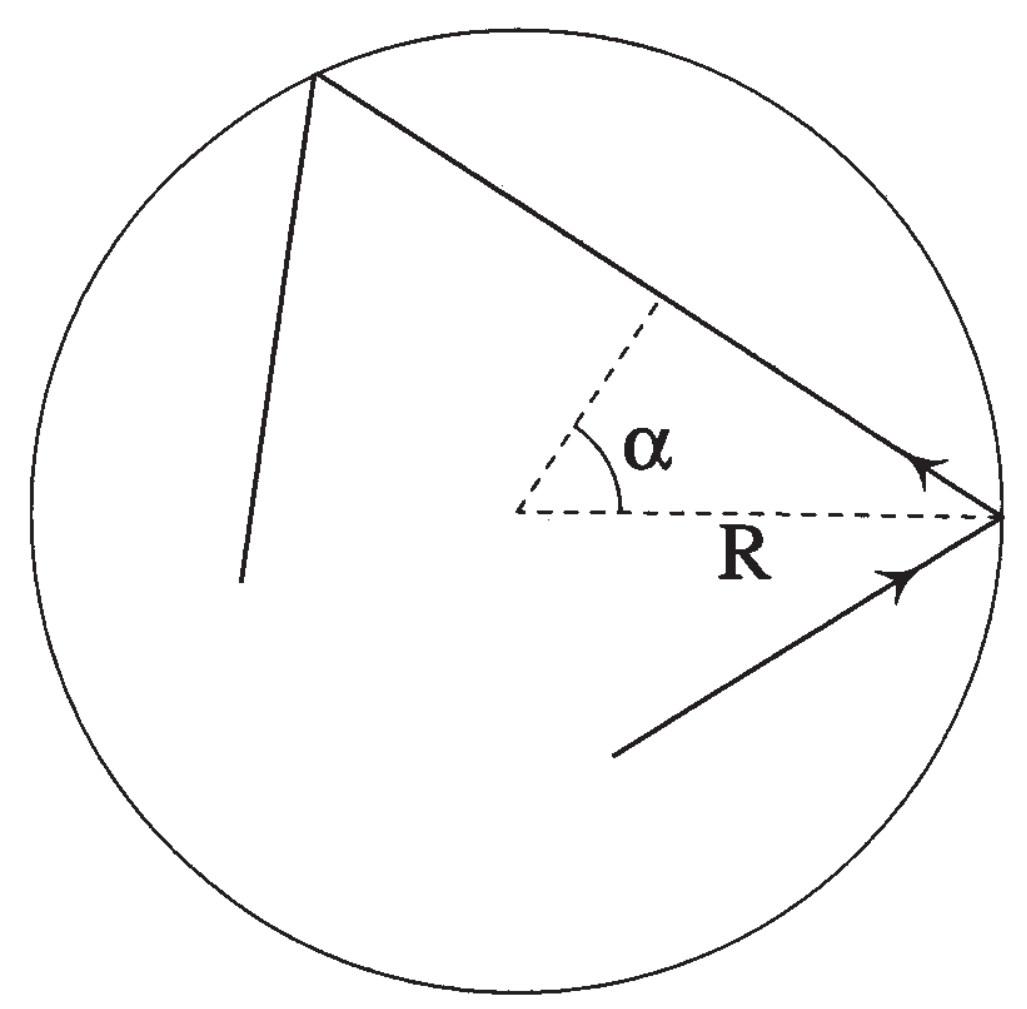

FIG. 1. Relation of angle $\alpha$ to nucleon trajectory in equilibrium mean field 


\section{TABLES}

TABLE I. Dynamic parameters for lowest multipolarities.

Columns a: present results, columns b: Ref.[7], columns c: model of Ref.[6]

\begin{tabular}{ccccccccc}
\hline \hline$L$ & $A_{L} / A_{0}$ & \multicolumn{3}{c}{$\gamma_{L} / \gamma_{w f}$} & \multicolumn{3}{c}{$D_{L} / m \varrho_{0} R^{5}$} \\
& $\mathrm{a}$ & $\mathrm{c}$ & $\mathrm{a}$ & $\mathrm{b}$ & $\mathrm{c}$ & $\mathrm{a}$ & $\mathrm{b}$ & $\mathrm{c}$ \\
\hline 0 & 1 & 0 & 0 & 0 & 0 & 0.2 & $\infty$ & - \\
1 & 0 & 0 & 0 & 0 & 0 & 1 & 1 & 1 \\
2 & 0.25 & 0 & 0 & 0 & 0 & $+\infty+i \frac{9}{32} \pi$ & $\infty$ & 0.5 \\
3 & 0 & 0 & 0.85 & 0.85 & 0 & 0.052 & 0.050 & 0.333 \\
\hline \hline
\end{tabular}

